\title{
Editorial: Topical Collection on Multi-Point Measurements of the Thermosphere with the QB50 Mission
}

\author{
Robert T. Wicks ${ }^{1}$. David M. Miles ${ }^{2}$
}

Published online: 4 February 2019

(C) Springer Nature B.V. 2019

The thermosphere is the top layer of the atmosphere, so named because temperature increases with altitude in this region. Starting at roughly $85 \mathrm{~km}$ altitude and rising until the edge of space is reached, the thermosphere is a low density $\left(\sim 10^{-12} \mathrm{~kg} / \mathrm{m}^{-3}\right.$ at $\left.400 \mathrm{~km}\right)$ gas in which constituent species behave separately since coupling of different mass population by collisions is negligible. It is the thermosphere in which solar UV radiation is absorbed to produce the conducting layers of the ionosphere and protect the ground from harmful UV light. Molecules of oxygen and nitrogen combine in chemical reactions to produce nitrous oxides of different compositions and charge states that are then spread around the world by winds of hundreds of kilometers an hour. These minor impurities change the chemistry of the thermosphere and so change the number of electrons produced by UV light in the ionosphere. These impacts change the temperature, density, and conductivity, of the thermosphere locally in ways that are not currently well modelled.

The thermosphere is also the region of the atmosphere first encountered by satellites and space debris that reach low Earth orbit. Drag on objects in orbit from the upper regions of the thermosphere is responsible for the de-orbiting of both space debris and functional satellites. Understanding and forecasting the density and temperature of the thermosphere is therefore important to predict the time and location of re-entry of objects into the Earth's lower atmosphere.

Measuring the properties of the upper layers of the atmosphere requires considerable effort. Observations from the ground can be made using radar, radio, or laser systems, but these are limited to studying a relatively small geographical region. Aircraft and balloons cannot generate lift in such tenuous atmosphere and so rockets are required to reach altitudes

Multi-Point Measurements of the Thermosphere with the QB50 Mission Edited by David Miles, Robert Wicks and James Burch

D.M. Miles

david-miles@uiowa.edu

1 Department of Space and Climate Physics, Mullard Space Science Laboratory, University College London, London, UK

2 Department of Physics and Astronomy, University of Iowa, Iowa City, IA, USA 
above $\sim 50 \mathrm{~km}$. Sounding rockets can be launched to provide vertical profiles of atmospheric conditions at one location at one time but provide, at most, a few minutes of data. Satellites that orbit at altitudes below $400 \mathrm{~km}$ are subject to large drag forces that make missions either very expensive in fuel or very short. These facts mean that there is a very limited amount of data on atmospheric conditions between $30 \mathrm{~km}$ and $300 \mathrm{~km}$ altitude. In particular, there are very few in-situ measurements that show the structure, winds, and composition of the global thermosphere. The QB50 mission is designed to provide the first multi-point measurements of the density, composition, and conductivity of the thermosphere using a distributed constellation of sensors on board CubeSats. The small size and low mass of CubeSats make them an ideal design for a low-cost constellation that can provide novel scientific data for a short period of time.

The QB50 mission aims to address several science questions relating to the thermosphere. First, how do the composition and density of the thermosphere vary in longitude, latitude, altitude and over time? Second, how do changing thermospheric conditions affect the drag on the satellites and how do the orbits of the CubeSats change in time? Finally, can we validate the remote sensing of the thermosphere using radar and laser systems as well as the numerical models of the thermosphere currently used for thermospheric research?

This Topical Collection provides the rationale and science goals for the QB50 mission and the summary of the diverse spacecraft which have been brought together to this end. It presents lessons learned from the coordination, control, and synthesis of data from thirty-six CubeSats provided by twenty-one different countries. The concept of CubeSat constellations is now popular but there are lessons to be learned from the QB50 project for similar future missions. This Topical Collection also documents the three common science payloads which the QB50 spacecraft hosted and how their data contribute to the mission's goals. The data from the three science instruments and the thermal sensors onboard each CubeSat launched as part of the mission is recorded and archived in the Von Karman Institute DPAC server and is available for future study. This volume also describes some of the different CubeSat designs that were used by the many different participants in the project. Each institution was able to design their own CubeSat along whatever lines they desired so long as the design met the mission requirements. Thus, there is a second unique data set collected from QB50 about the performance of different CubeSat designs.

Publisher's Note Springer Nature remains neutral with regard to jurisdictional claims in published maps and institutional affiliations. 